\title{
34. Note préliminaire sur la transformation de la peau larvaire en puparium chez les larves de Sturmia sericariae.
}

\author{
Par Soichi FuKuda. \\ Laboratoire séricicole de la Compagnie de Katakura, Matsumoto.
}

(Comm. by N. YATSU, M.I.A., April 12, 1937.)

On sait que les larves de Sturmia sericariae, après avoir quitté des corps de chrysalides de Vers à soie, rampent activement sans prendre aucune nourriture et enfin se métamorphosent en nymphes en tonnelet, changeant sa peau larvaire en puparium.

En été 1936, j'ai fait, pour commencer, une enquête sur l'échéance nécessaire à la transformation en pupae à partir de l'échappement des chrysalides. Pour cela, les larves qui sortent des chrysalides sont collectionnées chaque demi-heure dans des récipients et laissées passer le temps dans le laboratoire à la température de $23^{\circ}$ à $25^{\circ}, 5 \mathrm{C}$.

Tôt ou tard, toutes les larves se mettent à présenter l'indice de transformation en pupae. Telles larves s'arrondissent en arrière avec la peau plus ou moins dure et, par conséquent, roulent de côté, ne pouvant ramper en avant (cette condition se présente de 30 à 40 minutes avant le moment de devenir une pupa blanche). De plus en plus, elles sont forcées de se fixer à une place. Enfin, elles secouent seulement l'extrémité antérieure, le reste du corps se changeant en forme d'une partie de tonnelet immobile. Après de 5 à 10 minutes de cette condition la secousse du bout céphalique s'arrête et on trouve la pupa blanche un peu jaunâtre. Ce moment est registré comme le temps de transformation en puparium.

J'ai compté sur 1153 individus la durée nécessaire depuis l'échappement jusqu'à la transformation et obtenu les chiffres qui varient de 8,25 à 34 heures, la moyenne étant 19,08土0,07 heures.

La couleur de la pupa blanche ne tard pas à se changer, elle passe peu à peu au rougeâtre, puis au roussâtre et enfin au noir.

D'autre part, j'ai réalisé une série d'expériences de ligature des corps de larves à diverses époques, au moyen d'un fil de soie ou de coton. J'ai choisi les époques de larves comme les suivantes: immédiatement, 5,10 et 15 heures après l'échappement de chrysalides, de plus, de 30 à 40 minutes et de 5 à 10 minutes avant de devenir des pupae blanches. Le corps de larve est formé de douze segments. La ligature a été faite soineusement toujours à un niveau des sillons intersegmentaires. Donc il y a onze façons de ligature pour chaque époque des larves. Toutefois quant aux larves tout prêtes à devenir des pupae, il a été impossible de ligaturer en arrière.

Les résultats que j'ai obtenus ont été identiques, avec n'importe quel niveau de ligature, et même, à n'importe quelle époque des larves examinée. La partie antérieure reste à l'état tel quel, étant en vie pendant de 48 à 70 heures après la manipulation. Au contraire, la 
partie postérieure se modifie remarquablement; la peau devient dure avec la couleur roussâtre. $\mathrm{Au}$ cas même la ligature est faite au niveau de dernier sillon intersegmentaire la modification de la peau se produit seulement dans le dernier segment du corps (Fig. 1).

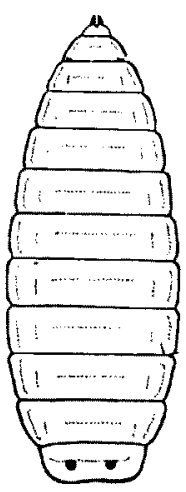

$\boldsymbol{a}$

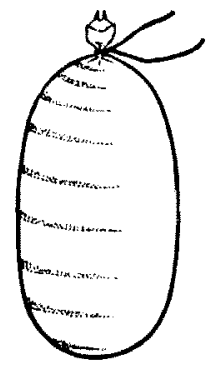

$b$

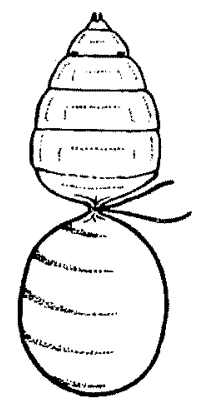

c

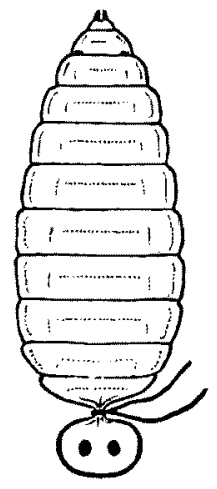

$d$

Fig. 1. Larves normale $(a)$ et ligaturées au moyen d'un fil (dessinées un peu schématique). La ligature est faite $b:$ au $2^{\mathrm{e}}, c$ : au $6^{\mathrm{e}}, d$ : au $11^{\text {e }}$ (le dernier) sillon intersegmentaire.

Ensuite, j'ai fait l'injection des liquides prélevés aux pupae blanches dans le corps des larves qui venaient d'échapper des chrysalides. La dose était de 0,07 à $0,08 \mathrm{cc}$ de l'humeur, qui a été employée avant qu'elle se changeât sa couleur. J'ai obtenu au total 230 individus traités et compté individuellement la durée depuis l'échappement jusqu'à la transformation, de la même façon que chez les larves non injectées.

Les effets d'injection de l'humeur ont été très nets. Les chiffres obtenus varient de 35 minutes à 10,83 heures avec la moyenne 3,57土 0,19 heures.

Avec l'espoir de provoquer artificiellement la transformation en puparium même à la partie antérieure de corps ligaturé, j'ai injecté de l'humeur provenante des pupae blanches dans cette partie. La dose était soit de $0,03 \mathrm{cc}$ soit de $0,08 \mathrm{cc}$. Le niveau de ligature était, dans ces cas, toujours au $8^{\mathrm{e}}$ ou $9^{\mathrm{e}}$ sillon intersegmentaire.

La partie antérieure, bien qu'elle reçoive les principes actifs, ne présente pas la modification caractéristique de la transformation en puparium. Cependant les larves sont en vie pendant de 15 à 18 heures après le traitement et la modification de la peau se produit dans la partie postérieure.

De ces résultats on peut supposer qu'il existe, à l'extrémité postérieure du corps, quelque chose d'indispensable pour déterminer la transformation de la peau larvaire en puparium et que c'est une paire des stigmates postérieurs.

Donc, j'ai réalisé une série d'expériences de fermeture des stigmates postérieurs, au moyen de la gélatine, chez les larves à diverses époques. Dans les cas des larves activement rampantes, elles bientôt cessent l'activité et enfin demeurent immobiles à l'état tel quel. Elles ne jamais présentent aucune indice de la transformation en puparium, néanmoins elles sont en vie pendant de 30 à 40 heures après la fermeture. 
Au cas même où les larves de 30 à 40 minutes avant de devenir des pupae blanches sont employées, elles aussi demeurent toujours à cet état tel quel. Enfin dans les cas des larves d'environs 5 minutes avant de la transformation en pupae, elles deviennent des pupae blanches presque normales, puisqu'elles cessent bientôt la secousse du bout céphalique, cependant la modification ultérieure n'est pas observée.

Finalement, j'ai effectué une série d'expériences de réouverture des stigmates postérieurs fermés. La fermeture a été pratiquée sur les larves à diverses époques. Les larves traitées sont laissées passer le temps durant $1,3,5,10,15,20$ et 30 heures, subséquemment la gélatine a été enlevée. J'ai obtenu sur 368 larves pratiquées au total 113 individus dont l'enlèvement de gélatine avait été bien réussi.

Dans tous les cas où la réouverture est bien réussie, les larves commencent à ramper de nouveau soit immédiatement soit après quelques dizaines de minutes et tôt ou tard se mettent à présenter la transformation en pupae. Enfin elles deviennent des pupae blanches normales, cependant chez les larves qui ont subi pendant longtemps la fermeture des stigmates, les pupae se déforment, sa couleur se changeant au roussâtre.

Il est important de noter ici s'il y a le raccourcissement de temps jusqu'à la transformation en pupae à la suite de la réouverture des stigmates. Tout du moins dans les cas observés je n'ai pas affirmé très remarquable raccourcissement de ce temps même après la longue échéance des stigmates fermés, par conséquent, on peut croire que la durée qui participe à la transformation en pupa a des relations intimes avec la période des stigmates postérieurs ouverts.

De l'ensemble de mes résultats expérimentaux, il est permis de tirer les conclusions suivantes. Le processus de la transformation de la peau larvaire en puparium chez les larves de Sturmia sericariae, après l'échappement des chrysalides de Vers à soie, est intimement lié à l'existence des stigmates postérieurs ouverts, qui ont peut-être à leur tour une relation avec le passage de l'oxygène atmosphérique. Il y a bien entendu quelques substances hormonales, dans les liquides provenants des pupae blanches, qui provoquent la transformation de la peau larvaire en puparium. Mais ces principes actifs, semble-t-il, ont besoin de plus, pour manifester leurs efficacités, de la coopération fonctionnelle des stigmates postérieurs ouverts.

A ma connaissance, la seule étude expérimentale sur la pupation des Diptères, est due à Fraenkel $(1935)^{1)}$ qui étudia chez les larves de Calliphora erythrocephala. Il a conclu l'existence de l'hormone qui amène la transformation en pupa. Cependant il n'a pas démontré la coopération des stigmates des larves durant le processus lui-même de la transformation en pupae.

1) Fraenkel, G. 1935 A hormone causing pupation in the blowfly Calliphora erythrocephala. Proc. Roy. Soc. London, Ser. B, 118, 1-12. 